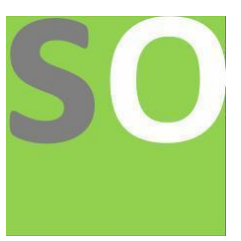

Article title: On the Interaction of the Electron with the Vacuum Fluctuations of Electromagnetic Field Authors: Xiaowen Tong[1]

Affiliations: China[1]

Orcid ids: 0000-0002-0389-8106[1]

Contact e-mail: yztsztxw@163.com

License information: This work has been published open access under Creative Commons Attribution License http://creativecommons.org/licenses/by/4.0/, which permits unrestricted use, distribution, and reproduction in any medium, provided the original work is properly cited. Conditions, terms of use and publishing policy can be found at https://www.scienceopen.com/.

Preprint statement: This article is a preprint and has not been peer-reviewed, under consideration and submitted to ScienceOpen Preprints for open peer review.

DOI: 10.14293/S2199-1006.1.SOR-.PP4NIOG.v1

Preprint first posted online: 04 February 2021

Keywords: interaction energy;, vacuum fluctuations, self-energy, Lamb shift, zero-point oscillations 


\title{
On the Interaction of the Electron with the Vacuum Fluctuations of Electromagnetic Field
}

\author{
Xiaowen Tong*
}

\begin{abstract}
A new approach is proposed for evaluating the interaction energy between the electron and the vacuum fluctuations of electromagnetic field. It is applied to two cases: when the electron is free and when it is in a potential-a hydrogen atom. The results are consistent with previous relevant treatments of people.
\end{abstract}

Unlike the energies of the states in which there are real photons being present, every mode of the zero-point energy posseesses a half of one quanta. Therefore, we guess that interaction energy of the electron interacting with the zero-point oscillations is precisely the half of the one between the electron and a state of radiation field of which every mode is occupied by one real photon(one photon for one polarization), which we call it the full one-photon state. Whereas the latter can be readily calculated as follows: Analogous to the self-action[1], the modification caused by the interaction to the wave function of the free electron is to multiply it a phase factor $\exp (-i \Delta E T)[1]$. The first-order of this modification corresponds to the process where only one photon is absorbed or emitted. We know that the S-matrix element of the Compton scattering in which the electron is scattered into the same eigenstate as its initial state is

$$
\begin{aligned}
M(k, \lambda)= & (-i e)^{2} \int d^{4} x_{1} d^{4} x_{2} \frac{m}{E} \frac{4 \pi}{2 \omega(2 \pi)^{3}} \bar{\psi}\left(x_{1}\right) \gamma_{\mu} i S_{F}\left(x_{1}-x_{2}\right) \gamma_{\nu} \psi\left(x_{2}\right) \\
& \cdot\left[\varepsilon_{\mu}(k, \lambda) \varepsilon_{\nu}(k, \lambda) e^{i k \cdot x_{1}} e^{-i k \cdot x_{2}}+\varepsilon_{\mu}(k, \lambda) \varepsilon_{\nu}(k, \lambda) e^{-i k \cdot x_{1}} e^{i k \cdot x_{2}}\right]
\end{aligned}
$$

Since there is one photon in every mode, we need to sum all the modes to get the modification of the wave function. Thus the interaction energy $\Delta E$ between the electron and the full one-photon state of radiation field is given by

$$
\lim _{T \rightarrow \infty}-i \Delta E T=\frac{1}{N} \sum_{\lambda=1}^{2} \int d^{3} k M(k, \lambda)
$$

In momentum space,

\footnotetext{
*The corresponding author: yztsztxw@163.com
} 


$$
\begin{aligned}
\Delta E= & 4 \pi e^{2} \frac{m}{E} \sum_{\lambda=1}^{2} \int \frac{d^{3} k}{(2 \pi)^{3}} \frac{1}{2 \omega_{k}} . \\
& {\left[\bar{u} \notin(k, \lambda) \frac{1}{\not p+\not k-m} \notin(k, \lambda) u+\bar{u} \notin(k, \lambda) \frac{1}{\not p-\not k-m} \notin(k, \lambda) u\right] }
\end{aligned}
$$

Using

$$
\not \not b=-\not \not \not d+2 a \cdot b, \quad \not^{2}=a^{2}
$$

we obtain

$$
\Delta E=\frac{4 \pi e^{2}}{E} \int \frac{d^{3} k}{(2 \pi)^{3}} \frac{1}{\omega_{k}}=\frac{4 \pi e^{2}}{E} \int \frac{d^{4} k}{(2 \pi)^{4}} \frac{2 i}{k^{2}+i \varepsilon}=\frac{m}{E} \frac{e^{2}}{2 \pi m} \lambda^{2}
$$

where in the last step we have used a direct cut-off(Wick rotation)[1]. Hence the energy shift of the electron caused by the fluctuations is

$$
\Delta E_{v f}=\frac{1}{2} \Delta E=\frac{m}{E} \frac{e^{2}}{4 \pi m} \lambda^{2} \stackrel{\text { nonrelativistic }}{\longrightarrow} \frac{e^{2}}{4 \pi m} \lambda^{2}
$$

This result is in accordance with previous treatments[2] ${ }^{*}$

Next we consider the Lamb shift which, as thought, is completely caused by the vacuum fluctuations of the radiation field. For simiplicity, we will derive it in nonrelativistic theory. The quantized transverse wave of electromagnetic field is

$$
\hat{\mathbf{A}}(\mathbf{x}, t)=\sum_{\mathbf{k}, \sigma} \sqrt{\frac{2 \pi \hbar c^{2}}{L^{3} \omega_{k}}} \varepsilon_{\mathbf{k} \sigma}\left(\hat{a}_{\mathbf{k} \sigma}(t) e^{i \mathbf{k} \cdot \mathbf{x}}+\hat{a}_{\mathbf{k} \sigma}^{\dagger}(t) e^{-i \mathbf{k} \cdot \mathbf{x}}\right)
$$

The interaction Hamiltonian is

$$
\hat{H}_{\mathrm{int}}=-\frac{e}{m c} \hat{\mathbf{p}} \cdot \hat{\mathbf{A}}(\mathbf{x}, t)+\frac{e^{2}}{2 m c^{2}} \hat{\mathbf{A}}^{2}(\mathbf{x}, t)
$$

Before calculating the Lamb shift, let us first evaluate the interaction energy for the free electron using our approach in nonrelativistic theory. We designate the state of the electron with momentum $\mathbf{p}$ and the full one-photon state as $|\mathbf{p}\rangle$ and $\left|1_{\text {full }}\right\rangle_{\text {rad }}$, repectively. According to our method, the second-order interaction energy is then

$$
\Delta E_{\mathbf{p}, v f}^{(2)}=\frac{1}{2}\left[\frac{e^{2}}{m^{2} c^{2}} \sum_{I} \frac{r_{a d}\left\langle 1_{f u l l}|\langle\mathbf{p}|\hat{\mathbf{p}} \cdot \hat{\mathbf{A}}| I\rangle\langle I|\hat{\mathbf{p}} \cdot \hat{\mathbf{A}}| \mathbf{p}\rangle| 1_{\text {full }}\right\rangle_{\text {rad }}}{E_{\mathbf{p}}-E_{I}}-\Delta E_{v a c}\right]
$$

*Our result is a half of Dalibard's. This is because the square of our cut-off momentum is the double of his. Weisskopf had also obtained this energy which, however, is incorrect[3]. He evaluated the mean square of the field fluctuations wrongly(Eq.(25)) which shall be one half of his value(for example, refer to[4]). He also calculated the integral incorrectly in Eq.(18) in his paper. 
where $\Delta E_{v a c}$ is the energy of the electron interacting with the vacuum which is given by

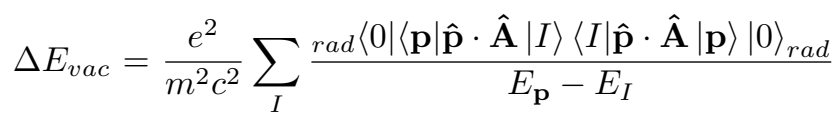

There is no this subtraction in the previous relativistic calculation for it has been implemented via the time-ordered product. We then have[5]

$\Delta E_{\mathbf{p}, v f}^{(2)}=\frac{1}{2} \frac{e^{2}}{m^{2} c^{2}} \frac{\hbar c^{2}}{(2 \pi)^{2}} \int \frac{k^{2} d k d(\cos \theta) d \phi}{k c}\left[\frac{(\hbar p)^{2}\left(1-\cos ^{2} \theta\right)}{-\hbar k c\left(1-\frac{\hbar p}{m c} \cos \theta+\frac{\hbar k c}{2 m c^{2}}\right)}+\frac{(\hbar p)^{2}\left(1-\cos ^{2} \theta\right)}{\hbar k c\left(1-\frac{\hbar p}{m c} \cos \theta-\frac{\hbar k c}{2 m c^{2}}\right)}\right]$

Being accurate enough for our case, the approximation $v / c \ll 1, \hbar \omega / m c^{2} \ll 1$ is applied to $\Delta E_{\mathbf{p}, v f}^{(2)}$. We obtain

$$
\Delta E_{\mathbf{p}, v f}^{(2)}=0
$$

Now we are ready to evaluate the Lamb shift:

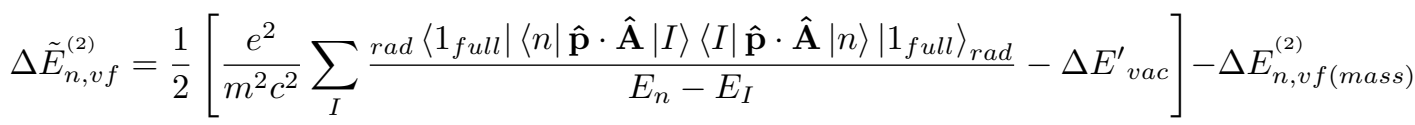

Here $|n\rangle$ is the eigenstate we considered of the hydrogen atom. The reason of the appearance of $\Delta E^{\prime}{ }_{v a c}$ is the same with $\Delta E_{v a c} . \Delta E_{n, v f(\text { mass })}^{(2)}$ is the correction to the energy corresponding to the variation of the electron mass. It equals to 0 in virtue of (4). Thus

$$
\begin{aligned}
& \Delta \tilde{E}_{n, v f}^{(2)}=\frac{1}{3 \pi} \frac{e^{2} \hbar}{m^{2} c} \sum_{n^{\prime}} \int_{0}^{\infty} k d k\left|\left\langle n^{\prime}|\hat{\mathbf{p}}| n\right\rangle\right|^{2}\left(\frac{1}{E_{n}-E_{n^{\prime}}-\hbar k c}+\frac{1}{E_{n}-E_{n^{\prime}}+\hbar k c}\right) \\
& =\frac{1}{3 \pi} \frac{e^{2}}{\hbar m^{2} c^{3}} \sum_{n^{\prime}}\left|\left\langle n^{\prime}|\hat{\mathbf{p}}| n\right\rangle\right|^{2}\left(E_{n^{\prime}}-E_{n}\right) \log \frac{\left(E_{n}-E_{n^{\prime}}\right)^{2}-\left(\hbar k_{c} c\right)^{2}}{\left(E_{n}-E_{n^{\prime}}\right)^{2}} \\
& \approx \frac{2}{3 \pi} \frac{e^{2}}{\hbar m^{2} c^{3}} \sum_{n^{\prime}}\left|\left\langle n^{\prime}|\hat{\mathbf{p}}| n\right\rangle\right|^{2}\left(E_{n^{\prime}}-E_{n}\right) \log \frac{m c^{2}}{\left|E_{n^{\prime}}-E_{n}\right|}
\end{aligned}
$$

The result is consistent with Dalibard's calculation[2].

The photons of the vacuum state of radiation field can not, of course, cause absorbtion transition of atoms. However, our discussion have shown that they can be considered as real ones to some degree. We may expect that the method will be valid as well in other processes envolving the energy for materials interacting with the vacuum fluctuations. The vacuum fluctuations are more "real" than we would think. 


\section{References}

[1] X. Tong, viXra: quant-ph/1812.0374

[2] J. Dalibard, J. Dupont-Roc and C. Cohen-Tannoudji, J. Physique 43 (1982) $1617-1638$

[3] V. F. Weisskopf, Phys. Rev. 56, 72, 1939

[4] T. A. Welton, Phys. Rev. 74, 1157, 1948

[5] Walter Greiner, Quantum Mechanics: Special Chapters: Section 5.2, Springer, 2004 